\title{
Analytical modelling of multi-mass flexible rocking structures
}

\author{
Sinan Acikgoz ${ }^{1, *,}$, Matthew J. DeJong ${ }^{1}$ \\ ${ }^{1}$ Department of Engineering, University of Cambridge, Cambridge, UK
}

\begin{abstract}
SUMMARY
This paper presents a new analytical model for describing the large rocking response of an elastic multi-mass structure resting on ideally rigid ground. Using the experimental results from a rocking steel column, the ability of the proposed analytical model to estimate the rocking and translational acceleration response under free vibration, pulse and earthquake excitations is evaluated. It is observed that the classical treatment of impact may result in an unrealistically large transfer of energy to vibrations. Therefore a new Dirac-delta type impact model that spreads the effects of impact over time and space is proposed. The use of a Diracdelta model and accurate restitution factors play a pivotal role in prediction of rocking and acceleration responses. In order to characterize the nonlinear response better, a modal analysis of the linearized system is proposed. With this approach, the vibration mode frequencies and shapes during rocking action were determined. A comparison of analytical and experimental modal estimations suggests good agreement. The results emphasize that the vibration characteristics of several vibration modes are affected by rocking action and these modes may be excited at impact.
\end{abstract}

KEY WORDS: rocking; uplift; impact; modal analysis

\section{INTRODUCTION}

The earthquake response of multi-mass flexible structures rocking on stiff ground is complicated, and modelling the response remains a challenge. Finite element methods are the preferred modelling tool, and have been successfully implemented for investigating various rocking structures in the past [1-3]. However, studies are typically structure-specific, consider calibrated damping models, and report various challenges in accurately capturing the rocking and vibration response [4-7]. In general, a better understanding of the dynamic behaviour of flexible rocking structures is important to improve both modelling and design, and to ensure safety. More specifically, a better fundamental understanding of (i) how superstructure vibration characteristics are affected during rocking and (ii) the ability of models to capture the concurrent energy loss and excitation of vibrations during impact, is necessary. An improved understanding of the vibration response will enable better prediction of the lateral force demand on the superstructure during rocking, and ensure that the intended base isolation provided by rocking can be utilised in design. A better modelling of impact will result in more reliable predictions of lateral displacements due to rocking.

Analytical models can be particularly useful in identifying fundamental dynamic behaviour. Pioneering studies by Psycharis [8] and Yim and Chopra [9] proposed complex analytical models to describe the dynamics of shear frames rocking on a viscoelastic two spring foundation. A general investigation of how vibration mode characteristics change during rocking concluded that only the first vibration mode interacts with rocking. They observed this interaction from two effects. First, the vibration frequency of the first vibration

\footnotetext{
* Correspondence to: Sinan Acikgoz, Department of Engineering, University of Cambridge, Trumpington Street, Cambridge, CB2 1PZ, UK

§ E-mail:msa44@cam.ac.uk
} 
mode increased once rocking initiated. Second, the vibrations were primarily excited during impacts with the ground, indicating the coupling between rotational accelerations due to rocking and translational accelerations due to vibrations. On the contrary, for all the other 'higher vibration modes', changes in modal frequencies during rocking and excitations due to impact were minimal. Therefore, it was concluded that the higher vibration modes are uncoupled from the rocking action. However, the analytical models proposed by Psycharis [8] and Yim and Chopra [9] have limitations. Both were derived for small rocking rotations, they did not consider inelastic energy dissipation due to impact and they evaluate structures deforming only in shear. More importantly, both models assumed the presence of classical orthogonal vibration modes during rocking action.

In this paper, a new analytical model is derived that addresses the mentioned limitations of previous models. The multi-mass model, introduced in Section 2, describes the nonlinear large rocking behaviour of an elastic superstructure. Different from Psycharis [8] and Yim and Chopra [9], a rigid ground is considered. However, a new impact model approximately accounts for the coupling of impact forces with superstructure vibrations. In Section 3, to understand the vibration response during rocking better, a method to decompose response into its modal components is presented. In Section 4, validation of the multi-mass model is carried out using results from a recent experimental campaign. First, the theoretical modal analysis is applied to the experimental model structure. This allows comparison of analytical and experimental estimations of modal components of response and leads to new insight regarding the interaction of rocking and vibration modes. Then, a comparison of nonlinear time history analyses and experimental tests follows. In particular, the effect of impact models on accurately modelling the experimentally observed rocking and lateral translational acceleration traces is evaluated. Correctly modelling these dynamic actions is essential as they relate closely to the lateral displacement and force demands experienced by the structure. Finally, Section 5 discusses primary fundamental findings and identifies effective modelling strategies.

\section{THE ANALYTICAL MODEL}

\subsection{Model parameters}

The multi-mass model that is proposed in this study is illustrated in Figure 1 during the full contact (left) and rocking (right) phases. The model is comprised of $N+1$ nodes, where masses are lumped. The foundation mass is partitioned between the two bottom nodes, Node 0 and Node 1 . Node 0 is located at $H_{0}=0$, with lumped mass $m_{0}$ and rotational inertia $J_{0}$. This node is restrained from participating in elastic motion. The remaining portion of the foundation mass is lumped at the adjoining Node 1, but this node is allowed participate in elastic motion due to finite foundation stiffness. For the remaining $N$ nodes (situated at height $H_{N}$ ), the mass is denoted by $m_{N}$ and represents a tributary portion of the mass of the superstructure. Therefore, for nodes 1 to $N$, the $N \times 1$ vector $\underline{H}$ describes nodal heights and the $N \times N$ diagonal mass matrix $\mathbf{M}$ describes the nodal masses.

The two degrees of freedom (DOF) associated with the small elastic lateral translation and rotation are illustrated by $u_{N}$ and $\Omega_{N}$ for node $N$ in Figure 1 . For nodes 1 to $N$, these DOFs can be described in vector notation as $\underline{u}$ and $\underline{\Omega}$. Typically, the connection between nodes was modelled with inextensible Euler beams with elasticity modulus $E_{s}$ and sectional moment of inertia $I_{s}$. However, the stiffness of the connection between the column and the foundation is not well represented by a beam element (see Section 4). Thus, at this location horizontal and rotational springs with stiffness $K_{f p}$ and $K_{r o t}$ were defined in parallel between 
nodes 0 and 1, as shown in Figure 1. The resulting full contact stiffness matrix $\mathbf{K}_{\mathrm{s}}$ has size $2 N \times 2 N$ and can be partitioned into four $N \times N$ matrices:

$$
\mathbf{K}_{\mathrm{s}}=\left[\begin{array}{ll}
\mathbf{K}_{\mathrm{tt}} & \mathbf{K}_{\mathrm{tr}} \\
\mathbf{K}_{\mathrm{rt}} & \mathbf{K}_{\mathrm{rr}}
\end{array}\right]
$$

where $\mathbf{K}_{\mathrm{tt}}$ describes the stiffness associated with $\underline{u}, \mathbf{K}_{\mathrm{rr}}$ describes the stiffness associated with $\underline{\Omega}$, and $\mathbf{K}_{\mathrm{tr}}$ and $\mathbf{K}_{\mathrm{rt}}$ are the stiffness coupling matrices. As will be described later, the stiffness matrix $\mathbf{K}_{\mathrm{s}}$ can be reduced to an $N \times N$ condensed stiffness matrix $\mathbf{K}$ by eliminating the rotational degrees of freedom (see Section 2.2).
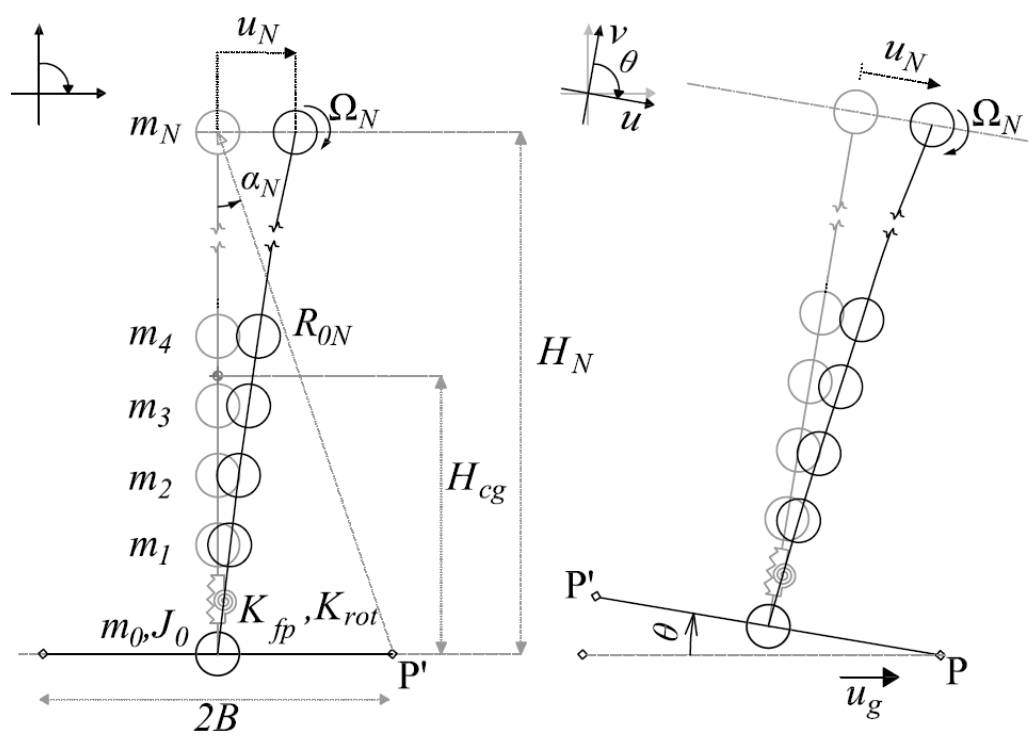

Figure 1. Schematic of the multi-mass analytical model during the full contact (top left) and rocking (top right) phases

During the rocking phase, the structure rotates about pivot points located at the edges of the foundation (see Figure 1, top row, right). The current pivot point is denoted by $\mathrm{P}$ and the impending pivot point location on the foundation beam is denoted by $\mathrm{P}^{\prime}$.These pivot points are a distance of $2 B$ apart. While the system is at rest, the radial distances from the current pivot point to each node are defined as $\underline{R_{0}}$. During rocking, the pivot point $\mathrm{P}$ is assumed to remain in contact with the ground at all times. The angle $\theta$ describes the rocking action.

To achieve linear equations of motion during full contact, inherent structural damping is modelled with Rayleigh damping, proportional to the full contact condensed mass and stiffness matrices. The formulations utilize the damping factors $\zeta_{n 1}$ and $\zeta_{n 2}$, which represent prescribed percentages of critical damping for the first two vibration modes. This results in the $N \times N$ proportional damping matrix $\mathbf{C}$. In this study, this matrix is also utilized to describe vibration damping during the rocking phase. Previous studies noted that this approach results in an increase in vibration damping during the rocking phase [8-10,13-17] and this increase may be unrealistic. Further exploration is the subject of continued research, but the current modelling approach led to a satisfactory comparison between experiments and analytical simulations (see Section 4). Nevertheless, refined proportional damping constants could be employed during the rocking phase if warranted by future experimental results. 


\subsection{Equations of motion}

The equations of motion of the multi-mass model under the influence of horizontal ground motion acceleration $\ddot{u}_{g}$ are defined in this section. The sign conventions used for the equations of motion of each phase are specified on the top left corners of the schematics in Figure 1.

Two sets of equations describe the dynamic equilibrium of the structure during the full contact phase. First, dynamic moment equilibrium about nodes 1 to $N$ yields:

$$
\mathbf{K}_{\mathrm{rt}} \underline{u}+\mathbf{K}_{\mathrm{rr}} \underline{\Omega}=\underline{0}
$$

This equation leads to the well-known static condensation equation, which allows the definition of a condensed stiffness matrix $\mathbf{K}=\mathbf{K}_{\mathrm{tt}}-\mathbf{K}_{\mathrm{tr}} \mathbf{K}_{\mathrm{rr}}{ }^{-1} \mathbf{K}_{\mathrm{rt}}$ [12]. Second, dynamic force equilibrium in the direction of $\underline{u}$ yields:

$$
\mathbf{M} \underline{\ddot{u}}+\mathbf{C} \underline{\dot{u}}+\mathbf{K} \underline{u}=-\mathbf{M} \ddot{u}_{g}
$$

where a dot above the variables indicates differentiation with respect to time $t$ and 1 specifies an $N \times 1$ vector of ones. Once conditions required for uplift are met, the system progresses to the rocking phase.

In the rocking phase formulations, large base rotations and small elastic deformations are considered. Three sets of equations describe the dynamic equilibrium: (i) $N$ equations describe dynamic moment equilibrium about nodes 1 to $N$, (ii) $N$ equations describe dynamic force equilibrium in the direction of $\underline{u}$ for nodes 1 to $N$ and (iii) one equation describes dynamic moment equilibrium about the current pivot point $P$. These equations are derived using a Lagrangian approach. The first set of equations is given by Equation (2). Then by replacing the system stiffness matrix with the condensed stiffness matrix, the second set of equilibrium equations can be expressed as:

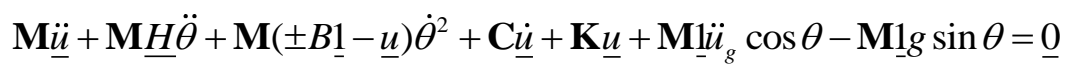

where $g$ denotes gravitational acceleration. In this equation the upper sign refers to rocking about the right pivot point and the lower sign refers to rocking about the left pivot point. A similar notation will be used in the current section and Section 3 to describe the piecewise defined equations of motion. The final equation of motion, describing the moment equilibrium about the pivot point, is:

$$
\begin{aligned}
& \underline{H}^{T} \mathbf{M} \underline{\ddot{u}}+\left(J_{\mathrm{P}}+\underline{u}^{T} \mathbf{M} \underline{u} \mp 2 B \underline{1}^{T} \mathbf{M} \underline{u}\right) \ddot{\theta} \mp 2 \underline{\dot{u}}^{T} \mathbf{M}(B \underline{1} \mp \underline{u}) \dot{\theta} \\
& +F_{i}(2 B)+\ddot{u}_{g}\left(\underline{H}^{T} \mathbf{M} \underline{1} \cos \theta \pm \underline{1}^{T} \mathbf{M}(B \underline{1} \mp \underline{u}) \sin \theta \pm m_{0} B \sin \theta\right) \\
& +g\left(-\underline{H}^{T} \mathbf{M} \underline{1} \sin \theta \pm \underline{1}^{T} \mathbf{M}(B \underline{1} \mp \underline{u}) \cos \theta \pm m_{0} B \cos \theta\right)=0
\end{aligned}
$$

where the system mass moment of inertia about the pivot point $\mathrm{P}$ is denoted by $J_{\mathrm{P}}=\underline{R_{0}}{ }^{T} \mathbf{M} \underline{R_{0}}+m_{0} B^{2}+J_{0}$. In this equation, the impact forcing term $F_{i}$ and its lever arm of

$2 B$ are introduced for the Dirac-delta impact model which is discussed in Section 2.4. The impact model makes use of an impact force which is defined to act perpendicular to the foundation beam at the impending pivot point $\mathrm{P}$ '. As the rocking structure approaches impact at $\theta=0$, the force $F_{i}$ is activated and dissipates energy by counteracting the motion (see Section 2.4).

Defining some additional parameters is useful for the presentation of results and characterization of the nonlinear response. Salient structural parameters include $H_{c g}, \alpha_{c g}$ 
and $p_{l}$, which are the height of the centre of gravity, the slenderness of the structure (with respect to the center of gravity) and the modified frequency parameter:

$$
p_{l}=\sqrt{\frac{\underline{1}^{T} \mathbf{M} \underline{H} g}{J_{\mathrm{P}}}}
$$

The modified frequency parameter provides a linear approximation of the pendulum frequency of the structure if it were suspended from its pivot point, and is an important parameter with respect to the rotational response. In addition, the acceleration vector $\underline{a}$ is defined as the total acceleration in the direction of $\underline{u}$ for nodes $1-N$ as:

$$
\underline{a}=\underline{\ddot{u}}+\underline{H} \ddot{\theta}+( \pm B \underline{1}-\underline{u}) \dot{\theta}^{2}+\underline{u ̈}_{g} \cos \theta-\underline{1} g \sin \theta
$$

\subsection{Phase transition}

In the formulation presented in Section 2.2, the structure alternates between the respective full contact and rocking phases. The conditions for the transitions between these phases are discussed in this section.

For the structure to proceed from a full contact phase to a rocking phase, two conditions need to be met. First, $M_{o}^{f c}$, the overturning moment about the impending pivot point must exceed the restraining moment $M_{\text {res }}^{f c}$ about the same corner. Second, angular momentum $L_{\mathrm{P}^{\prime}}^{f c}$ about the impending pivot point should be in the direction of impending rocking motion. The first condition alone signifies uplift, but the second condition is also suggested to ensure numerical stability. They can be summarized as follows:

$$
\mp M_{o}^{f c}>M_{r e s}^{f c}, \pm L_{\mathrm{P}^{\prime}}^{f c}>0
$$

where the upper signs define impeding rocking about the right pivot point and the lower signs define impending rocking about the left pivot point. This notation is consistently used to describe phase transitions and impact models throughout Sections 2.3 and 2.4.

Equations (8) are evaluated at every time step during the full contact phase and upon satisfying these conditions, a rocking phase is initiated. The elastic displacements and velocities remain the same during this transition. Therefore, the post-transition parameters, which will be used as initial values for the new rocking phase, are given as $\dot{\theta}^{+}=\dot{\theta}^{-}=0$ and $\underline{\dot{u}}^{+}=\underline{\dot{u}}^{-}$where the superscripts ${ }^{-}$and ${ }^{+}$denote the pre- and post-transition parameters.

The simulation of a rocking half-cycle ends when $\theta=0$. At this moment of phase transition, a new rocking half-cycle may be initiated or the structure may proceed to a full contact phase. Two conditions are used to determine the next phase of motion:

$$
\pm L_{\mathrm{P}^{\prime}}^{r} \geq 0, \pm \dot{\theta}^{-}>\dot{\theta}_{\text {min }}
$$

The first condition evaluates the angular momentum about the impending pivot corner $L_{\mathrm{p}}^{r}$. The angular momentum condition in Equation (9) intuitively prescribes that for a new rocking phase to begin, the total angular momentum of the structure about the impacting corner should be in the same direction as the angular velocity of the base at the instant of impact. The second condition sets a minimum angular velocity $\dot{\theta}_{\min }$ to ensure numerical stability. If small values are specified for $\dot{\theta}_{\min }$, the simulation takes longer due to smaller integration time step requirements. In this paper, $\dot{\theta}_{\min }=0.01 \mathrm{rad} / \mathrm{s}$ is utilised. If either of these conditions in Equation (9) is not satisfied, then a full contact phase is assumed to begin. 
The complex transition between phases is typically simplified with intuitive but unvalidated conservation rules [8-9, 13-14]. A similar approach is initially followed in this study, but these assumptions are assessed critically later. When a transition from a rocking phase to a full contact phase occurs, it is assumed that the vertical momentum is completely dissipated, while the horizontal momentum at each node is conserved. This results in an energy loss and a non-smooth transition:

$$
\dot{\theta}^{+}=0, \underline{\dot{u}}^{+}=\underline{\dot{u}}^{-}+\underline{H} \dot{\theta}^{-}
$$

However, if the conditions presented in Equations (9) are satisfied, then a new rocking phase is initiated about the opposing pivot point. The post-transition parameters obtained for each impact model are different. For the classical impact model, energy is dissipated in the instantaneous non-smooth transition. The post-transition parameters are assumed as:

$$
\dot{\theta}^{+}=r \dot{\theta}^{-}, \dot{u}^{+}=\dot{u}^{-}
$$

where $r$ denotes the coefficient of restitution. In this transition, similar to earlier studies (e.g. [14]) the elastic translational velocities are assumed to remain the same while the angular velocity is reduced. Conversely, for the Dirac-delta impact model (see Section 2.4), it is not necessary to make unvalidated assumptions concerning post-impact parameters. Here, energy is dissipated gradually by applying an impact force which lasts for a finite duration just prior to the rocking angle reaching zero. Then, when $\theta=0$, a transition to a new rocking phase occurs smoothly. Initial parameters for the new rocking phase are simply given by:

\subsection{Impact models}

$$
\dot{\theta}^{+}=\dot{\theta}^{-}, \dot{u}^{+}=\dot{u}^{-}
$$

Two impact models are considered for the transition from one rocking phase to another: the classical model and the Dirac-delta model. The first model assumes a classical impact framework, where the duration of impact is assumed to be infinitesimally small. Conversely, the second method models impact forces by an approximation to the Dirac-Delta functional by a zero-centred Gaussian function, similar to the one used by Prieto et al. [19].

In the classical impact model, impact effects are assumed to occur instantaneously at $\theta=0$. Due to this assumption of infinitesimal duration, the translational velocities are assumed to remain the same while the angular velocity is reduced using Equation (11). If the impact forces are concentrated at the impacting corner, conservation of angular momentum about this corner results in an approximation of $r$, denoted by $r_{h}$ :

$$
r_{h}=\frac{\dot{\theta}^{+}}{\dot{\theta}^{-}}=\frac{J_{\mathrm{P}}-2 m_{t} B^{2}+\underline{u}^{T} \mathbf{M} \underline{u}}{J_{\mathrm{P}} \pm 2 B\left(\underline{1}^{T} \mathbf{M} \underline{u}\right)+\underline{u}^{T} \mathbf{M} \underline{u}}
$$

Equation (13) can be simplified by neglecting elastic displacements, resulting in an estimate of $r_{h}$ based only on geometric characteristics of the structure. Previous studies (e.g. [18]) suggest that $r_{h}$ may provide an unrealistically large estimate of the energy dissipated during motion for stocky structures where $\alpha_{c g}>0.2$. Therefore, when available, the use of empirical coefficients of restitution $r_{e}$ should be preferred. The energy loss in this impact model can be approximated as $E_{i n i}\left(1-r^{2}\right)$, where $E_{i n i}$ is the total system energy at the moment of impact initiation.

As an alternative approach, the new Dirac-delta model considers the interaction of impact forces and superstructure vibrations. In this model, as the structure approaches $\theta=0$, an impact force $F_{i}$ (see Equation (5)) act on the rigid foundation beam at the impending pivot 
point $\mathrm{P}^{\prime}$. This force applies moments counteracting the rocking, resulting in rotational decelerations that influence the elastic motion of the structure during the impact. After the phase transition at $\theta=0$ (governed by Equation 12), the force is terminated as any forcing from the pivot point will no longer influence the moment equilibrium after Stage $\mathrm{V}$. This process is schematically illustrated in Figure 2 (top row).

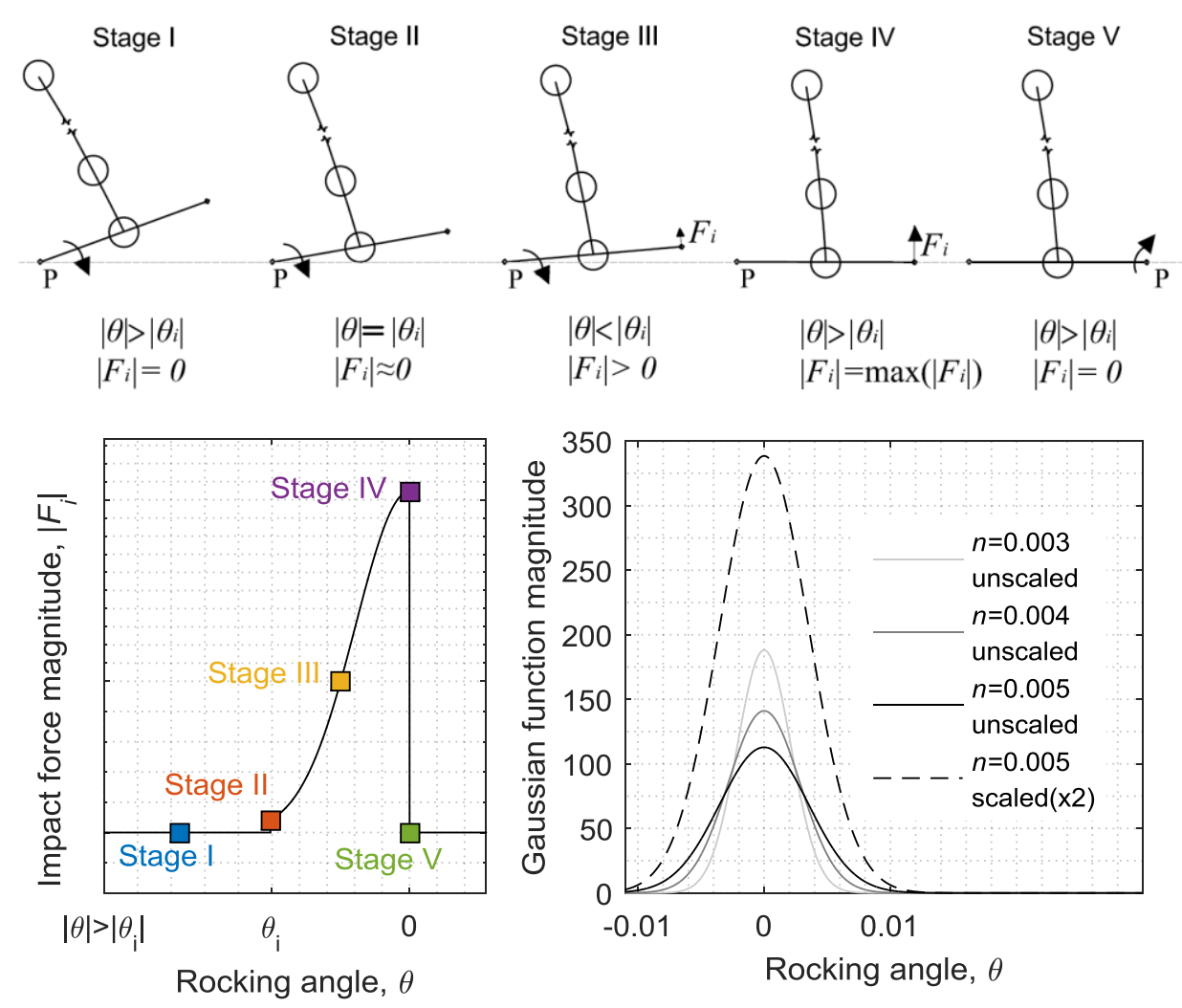

Figure 2. Schematic illustration of the applied impact forces for the Dirac-Delta model (top row), variation of impact force magnitude with the rocking angle (bottom row, left), and the effect of various parameters on the shape of the impact force (bottom row, right).

The impact force $F_{i}$ is approximated by an amplitude scaled Gaussian function of the rocking angle. The Gaussian function is defined by a zero mean and a standard deviation of $n / \sqrt{2}$. Here, $n$ is a width parameter of the Gaussian function that influences the sharpness of the impact force. The effect of changing the value of $n$ on the function form is illustrated in Figure 2 (bottom row, right). Independent of the value of $n$, when the unscaled Gaussian function is integrated over values of $\theta$ in the domain $(-\infty,+\infty)$, a value of 1 is obtained. Using this property, the amplitude of the Gaussian function can be scaled to cause an energy loss of $E_{i n i}\left(1-r^{2}\right)$. Then the following expression emerges for $F_{i}$ :

$$
F_{i}= \pm \frac{E_{i n i}\left(1-r^{2}\right)}{B n \sqrt{\pi}} e^{-\theta^{2} / n^{2}}
$$

A physically motivated approach is necessary for determining the width parameter $n$. It is postulated that the maximum impact force would be proportional to the angular velocity of the structure at the moment impact forces are activated (denoted by $\dot{\theta}_{i}$ ). Additionally, considering the limit case of zero angular velocity, the maximum impact force on the corner can be considered bounded by the static support force at rest position, which is $m_{t} g / 2$. Here, $m_{t}$ denotes the total mass. These assumptions result in the following expression: 


$$
n=\frac{E_{\text {ini }}\left(1-r^{2}\right)}{B \sqrt{\pi}\left(\frac{m_{t} g}{2}+C_{p}\left|\dot{\theta}_{i}\right|\right)}
$$

where $C_{p}$ is a proportionality constant which describes the relationship between $\dot{\theta}_{i}$ and $F_{i}$. Since this term relates to the mass and stiffness of the specific impacting surfaces, an empirical value for $C_{p}$ that produces the best fit with the experimental results is determined in Section 4.

Equation (15) depends on the system state at the initiation of impact forces, as described by parameters $E_{i n i}$ and $\dot{\theta}_{i}$. Therefore, at every time step during the simulation of a rocking phase, conditions governing the initiation of the impact force need to be checked:

$$
\pm \theta> \pm \theta_{i}=\mp 2.576(n / \sqrt{2}), \quad \pm L_{\mathrm{P}^{\prime}}^{r}>0, \quad \pm \dot{\theta}_{i}>\dot{\theta}_{\text {min }}
$$

The first condition in Equations (16) ensures that the impact forces are only activated at small rocking angles when the structure approaches impact. As a result, the impact forces do not interfere with large rocking motion. In particular, the coefficient 2.576 in the first condition, describes the $99.5 \%$ confidence interval of the Gaussian function. This coefficient ensures that the specified amount of energy is dissipated when the impact forces are active. The following two conditions are identical to phase transition conditions presented in Equations (9) and follow a similar rationale.

In the case that any of the conditions in Equation (16) is not met, the impact forces are not activated. Then, at every time step, $n$ and $\theta_{i}$ are recalculated using the current system state and conditions in Equations (16) are evaluated. When these conditions are satisfied at a particular time step, the specific values of $E_{i n i}, \theta_{i}$ and $\dot{\theta}_{i}$ are fixed. These values are used to compute an impact-specific $n$ value using Equation (15). In general, smaller values of $n$ are obtained for smaller rocking motion, resulting in sharper impact forces and smaller values for $\theta_{i}$ (see Figure 2). Until the impact force is deactivated, these constant parameters are used alongside the current value of $\theta$ in the calculation of $F_{i}$ with Equation (14). To deactivate the impact forces, one of the following conditions needs to be satisfied:

$$
\pm \theta< \pm 1.1 \theta_{i}, \quad \theta=0
$$

The first condition deactivates the forces when the system moves away from impact shortly after the initiation of forces. A constant coefficient of 1.1 has been arbitrarily specified to define this phenomenon. With reference to Figure 2, this case would imply that impact forces are activated at Stage 2, but then the structure moves back towards Stage 1, suggesting that impact is not imminent and forces should be deactivated. The second condition describes the end of a rocking half-cycle. Here, transition to a new full contact or rocking phase is determined (see Section 2.3).

The equations described above were numerically solved in MATLAB using implicit and explicit ODE solvers [20]. The stiff nature of the equations of motion resulted in the use of maximum time step limits. For computational efficiency, a minimum value of $\min (n)=0.0001$ was additionally specified.

\section{MODAL DECOMPOSITION}

In order to characterize the nonlinear response of the multi-mass analytical model described in Section 2, the current section describes a method to determine the modal components of response. Instead of assuming that higher vibration modes are uncoupled ([8-9]), this method 
investigates the coupling of all vibration modes with rocking action. Initially, a first-order approximation is made to the nonlinear equations of motion of the multi-mass system during the rocking phase (described by Equations (4-5)). Using the formal linearization procedure outlined in [21], linearization about the static rest configuration $(\theta=0, \dot{\theta}=0, \underline{u}=\underline{0}$ and $\underline{\dot{u}}=\underline{0})$ yields:

$$
\begin{aligned}
& \mathbf{M} \underline{\ddot{u}}+\mathbf{M} \underline{H} \ddot{\theta}+\mathbf{C} \underline{\dot{u}}+\mathbf{K} \underline{u}-\mathbf{M} \underline{1} g \theta+\mathbf{M} \underline{1} \ddot{i} g=\underline{0} \\
& \underline{H}^{T} \mathbf{M} \underline{\ddot{u}}+J_{\mathrm{p}} \ddot{\theta}+g\left(-\underline{u}^{T} \mathbf{M} \underline{1}-\underline{H}^{T} \mathbf{M} \underline{1} \theta\right)+m_{t} H_{c g} \ddot{u}_{g} \pm m_{t} g B=0
\end{aligned}
$$

The linearized equations result in a set of second order differential equations with constant coefficients. Therefore, Equations (19) and (20) may be expressed in the following format:

$$
\mathbf{M}_{\mathbf{L}} \underline{\ddot{z}}+\mathbf{C}_{\mathbf{L}} \underline{\dot{z}}+\mathbf{K}_{\mathbf{L}} \underline{z}=-\underline{F_{L}}
$$

where the state vector $\underline{z}=[\underline{u}, \theta]$ encompasses all the variables in the system. The resulting set of second order differential equations can be expressed as a classical eigenvalue problem when the Caughey O'Kelly condition [22] is satisfied. Before rocking initiates, this condition is satisfied due to the assumption of proportional damping. This suggests that the eigenvectors of the system can be readily identified using the undamped system characteristics. However, once rocking initiates, the Caughey O'Kelly condition is not satisfied. Therefore, the eigenvectors defined by using an undamped configuration will not provide a precise definition of system vibration characteristics. However, a different methodology can be followed to determine the modal parameters. First, by using Rayleigh's method, the state vector may be expressed as follows:

$$
\underline{z}=\mathbf{v}_{\mathbf{u}} \underline{q_{u}}
$$

where $v_{\mathbf{u}}=\left[\underline{v_{u 1}}, \underline{v_{u 2}}, \ldots, \underline{v_{u N+1}}\right]$ is the $(N+1) \times(N+1)$ matrix of rocking phase eigenvectors. The $j^{\text {th }}$ column of $\boldsymbol{v}_{\mathbf{u}}$ describes the $j^{\text {th }}$ eigenvector of the system, denoted by $v_{u j}$. Furthermore, this vector can be expressed as $\underline{v_{u j}}=\left(\underline{v_{u j t}}, v_{u j r}\right)^{T}$ where $\underline{v_{u j t}}$ is the elastic eigenvector component in the direction of $u$ and $v_{u j r}$ is the rocking component which describes the modal rigid body rotation of the structure as a whole. The generalized coordinate vector $\underline{q_{u}}$ describes the corresponding modal solutions as follows:

$$
\underline{q_{u}}=\left(q_{u 1}, q_{u 2}, \ldots ., q_{u N}, q_{u N+1}\right)^{T}=\left(\varepsilon_{u 1} e^{\Lambda_{u 1} t}, \varepsilon_{u 2} e^{\Lambda_{u 2} t}, \ldots . ., \varepsilon_{u N} e^{\Lambda_{u N} t}, \varepsilon_{u N+1} e^{\Lambda_{u N+1} t}\right)^{T}
$$

where $\varepsilon_{u j}$ is an arbitrary scale factor and $\Lambda_{u j}$ denotes the eigenvalue of the $j^{\text {th }}$ normal mode of the system. Then, to perform modal analysis on the non-classically damped system, the system velocities need to be expressed as separate dependent variables using Foss' method [23]. Then, Equation (21) can be expressed as the following eigenvalue problem:

$$
\Lambda_{u j}\left(\begin{array}{c}
\frac{v_{u j}}{\Lambda_{u j} v_{u j}}
\end{array}\right)=\left[\begin{array}{cc}
\mathbf{0} & \mathbf{I} \\
-\mathbf{M}_{\mathbf{L}}^{-1} \mathbf{K}_{\mathbf{L}} & -\mathbf{M}_{\mathbf{L}}^{-1} \mathbf{C}_{\mathbf{L}}
\end{array}\right]\left(\begin{array}{c}
\frac{v_{u j}}{\Lambda_{u j} v_{u j}}
\end{array}\right)
$$


where $\mathbf{I}$ is the $(N+1) \times(N+1)$ identity matrix. Solving Equation (24) results in $2 N+2$ eigenvalues. As eigenvalues appear in complex conjugate couples, the resulting set describes $N+1$ modes. Correspondingly, eigenvectors of modes appear as complex conjugate couples. The modal frequency $\omega_{u k}$ and damping $\zeta_{u k}$ of the uplifted vibration modes are:

$$
\omega_{u k}=\left|\Lambda_{u k}\right|, \zeta_{u k}=-\frac{\operatorname{Re}\left(\Lambda_{u k}\right)}{\omega_{u k}}
$$

where $k=1, \ldots, N$. The operators $\operatorname{Re}$ and $\operatorname{Im}$ (see Figure 4 later) denote the real and imaginary parts of a complex number, and $\mid$ denotes the modulus of a complex number.
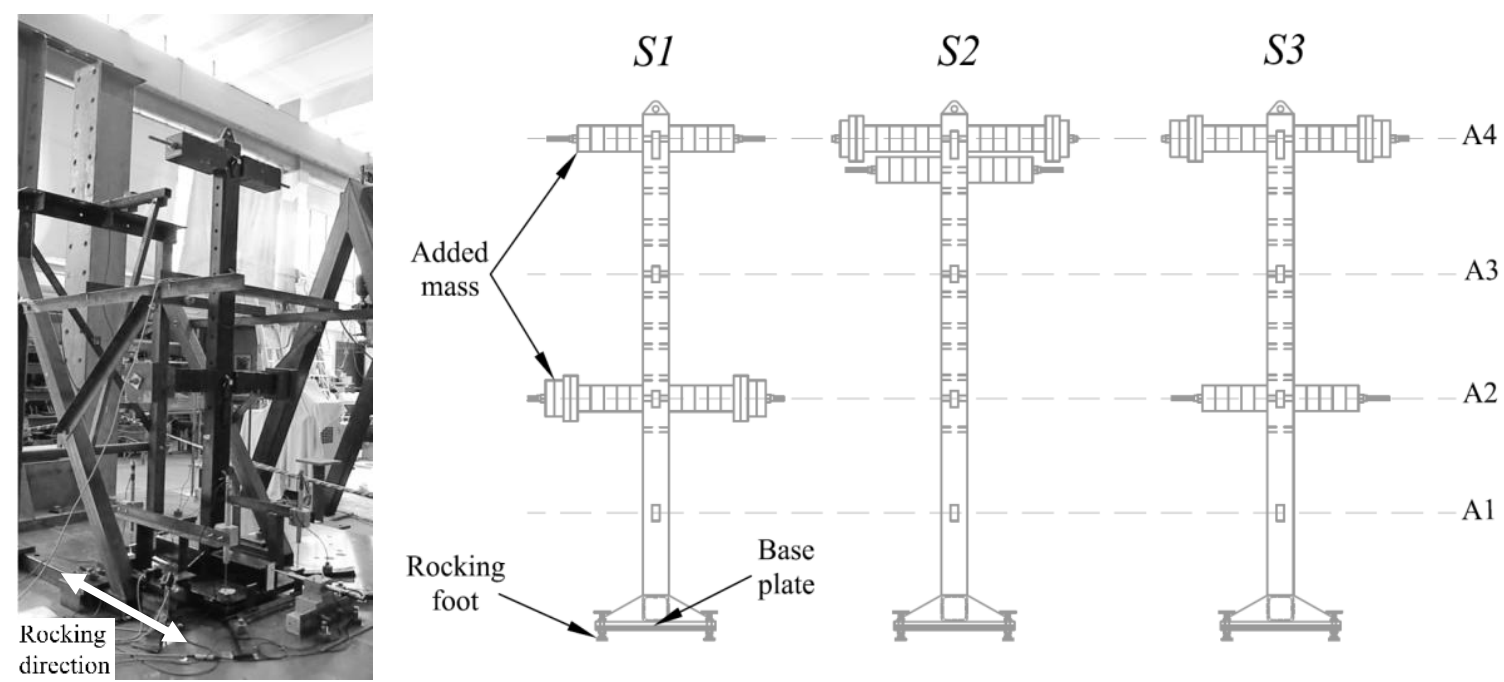

Figure 3. (Left) A photo and (right) elevation drawings of the experimental model illustrating the location of the accelerometers A1-A4 and the mass arrangements S1, S2 and S3.

\section{EVALUATION OF ANALYTICAL MODELS}

In this section, experimental tests which are used to validate the analytical models are introduced. Then, analytical models of the experimental specimens, created using the formulations in Section 2, are presented. The modal decomposition approach from Section 3 is evaluated through comparison with experimental results, and general conclusion are drawn regarding the vibration mode characteristics during rocking. Finally, the accuracy of the multi-mass model in simulating rocking and translational acceleration response is evaluated by comparing nonlinear response simulations and experimental free vibration, pulse and earthquake tests.

\subsection{Analytical models of experimental specimens}

Figure 3 shows a photo and drawings of the experimental model which is comprised of an approximately $2 \mathrm{~m}$ tall rectangular hollow steel section welded on an approximately $30 \mathrm{~mm}$ thick and laterally stiffened base plate. The plate is supported by four "feet" at its corners, which rest on the shake table. Each foot is comprised of an M16 bolt, screwed into the base plate and extending $40 \mathrm{~mm}$ beneath the base plate, which is welded to a $40 \mathrm{~mm}$ wide and $5 \mathrm{~mm}$ thick square plate, which provides contact with the shake table. Masses were attached along the height of the column to simulate structures with different geometry and vibration characteristics. Three mass arrangements/specimens were tested, denoted by S1, S2 and S3. The experimental model was instrumented with displacement sensors to measure the rocking 
angle and accelerometers to measure the translational lateral accelerations along the height of the structure. Free vibration, pulse and earthquake tests were carried out on each specimen. Relevant information regarding the experiments are discussed in the text but further details can be found in [11].

Table 1. Input parameters for the multi-mass structural models of the three experimental specimens. Common parameters utilized for all models are listed under the table.

\begin{tabular}{|c|c|c|c|}
\hline Parameters & S1 & S2 & S3 \\
\hline$\underline{H}(\mathrm{~m})$ & $\begin{array}{c}{[0.06,0.49,0.93} \\
1.43,1.93]^{T}\end{array}$ & $\begin{array}{c}{[0.06,0.49,0.93} \\
1.43,1.89]^{T}\end{array}$ & $\begin{array}{c}{[0.06,0.49,0.93,} \\
1.43,1.93]^{T}\end{array}$ \\
\hline $\operatorname{diag}(\mathbf{M})(\mathrm{kg})$ & $\begin{array}{c}{[77.3,6.2,88.2} \\
8.2,46.2]^{T}\end{array}$ & $\begin{array}{c}{[77.3,6.2,7.7} \\
7.9,123.1]^{T}\end{array}$ & $\begin{array}{c}{[77.3,6.2,48.1} \\
8.2,86.2]^{T}\end{array}$ \\
\hline$m_{0}(\mathrm{~kg})$ & 0 & 0 & 0 \\
\hline$J_{0}\left(\mathrm{kgm}^{2}\right)$ & 10 & 10 & 10 \\
\hline$J_{\mathrm{p}}\left(\mathrm{kgm}^{2}\right)$ & 298 & 509 & 413 \\
\hline$H_{c g}(\mathrm{~m})$ & 0.84 & 1.18 & 1.02 \\
\hline$\alpha_{c g}(\mathrm{rad})$ & 0.35 & 0.25 & 0.29 \\
\hline$p_{l}(\mathrm{rad} / \mathrm{s})$ & 2.51 & 2.26 & 2.35 \\
\hline$\left(\zeta_{n 1}, \zeta_{n 2}\right)(\%)$ & $0.005,0.015$ & $0.0025,0.015$ & $0.005,0.015$ \\
\hline$\left(\overline{r_{e r}}, \overline{r_{e l}}\right)(-)$ & $0.93,0.87$ & $0.98,0.93$ & $0.94,0.91$ \\
\hline$\sigma_{r}(-)$ & 0.025 & 0.0175 & 0.025 \\
\hline
\end{tabular}

\subsection{Modal components of response}

Modal analyses were carried out by solving the eigenvalue problem in Equation (24). As an example, Figure 4 shows the solutions for S1. The eigenvalues were evaluated for the damped configuration during the full contact and rocking phases and are plotted on the complex plane. Meanwhile, the eigenvectors were evaluated numerically for the undamped configuration to obtain real-valued eigenvectors. These do not differ significantly from the complex eigenvectors computed for the damped configuration.

To represent the eigenvectors, only nodes 2-5 were considered to facilitate comparison to experimental results. The designated nodal values for the 'full contact phase eigenvector' $v_{n j}$ and elastic component of the corresponding rocking phase eigenvector $v_{u j t}$ are scaled to Euclidean norm of unity and are presented in the figure for $j=1,2,3, r$. The rocking action associated to mode $j$, represented by the appropriately scaled $\underline{v_{u j r}}$, was later superposed on the elastic mode shape $\underline{v_{u j t}}$ to find the compound shape as $\underline{v_{u j c}}=\underline{v_{u j t}}+\underline{H} v_{u j r}$. Under each vibration mode eigenvector, the associated modal frequencies before and during rocking motion are presented respectively in brackets as $\left[\omega_{n k}, \omega_{u k}\right]$ for $k=1,2,3$.

In Figure 4 (left), the complex eigenvalue couples describe the $1^{\text {st }}$ and $2^{\text {nd }}$ vibration modes of the structure during the full contact and rocking phases. Interestingly, the eigenvalue of the $1^{\text {st }}$ vibration mode indicates a threefold increase in frequency with the initiation of rocking from $7.6 \mathrm{~Hz}$ to $20.7 \mathrm{~Hz}$. For the $2^{\text {nd }}$ vibration mode, a smaller increase is observed from $41.4 \mathrm{~Hz}$ to $46.9 \mathrm{~Hz}$. A similar increase is observed for the modal damping of both of these vibration modes. These changes suggest coupling between rocking and vibrations for these two modes. Additionally, the symmetric eigenvalue couple lying on the real axis of Figure 4 (left) describes the rocking mode. The quantity $\pm p_{l}$ in Equation (6), 
provides an excellent estimation of this eigenvalue. This indicates that the motion of the rocking mode is largely determined by the geometric characteristics of the structure.

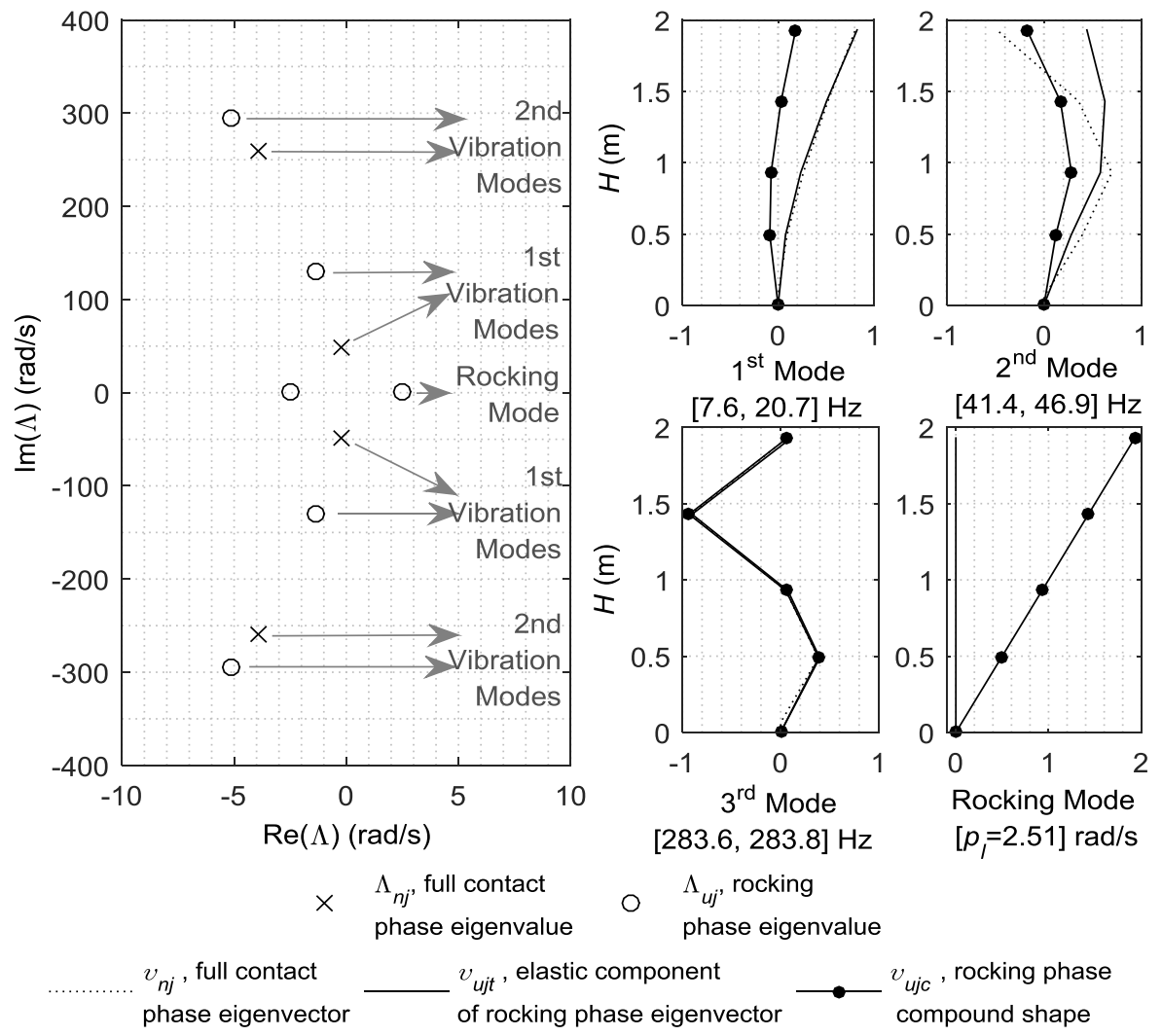

Figure 4. Linearized system damped configuration eigenvalues (left) and undamped configuration eigenvectors (right) for S1 during full contact and rocking phases. Under each mode eigenvector, the estimates for modal frequencies in these phases are respectively shown in brackets.

Comparing the first vibration mode eigenvector during the full contact and rocking phases provides further insight, see Figure 4 (top row, middle). The elastic component of the vibration mode shape during rocking the phase, $v_{u 1 t}$, is not altered significantly from the vibration mode shape during the full contact phase, $v_{n 1}$. However, during rocking, base rotation is induced by the superstructure vibrations. When the effect of this oscillatory rocking component is considered, a drastically different compound shape, $\underline{v}_{u 1 c}=\underline{v_{u 1 t}}+\underline{H} v_{u 1 r}$, is obtained. A nodal point is now observed at $H \approx 1.3 \mathrm{~m}$, where eigenvector ordinates change polarity. In contrast, the compound shape of the second vibration mode during the rocking phase $\underline{v}_{u 2 c}$ is more similar to the full contact phase eigenvector $\underline{v_{n 2}}$. However, the vibration mode shapes which only explore elastic components of this vibration mode, $v_{n 2}$ and $\underline{v_{u 2 t}}$, are quite different, verifying that the $2^{\text {nd }}$ mode is also coupled with rocking. Meanwhile, the $3^{\text {rd }}$ mode eigenvector remains nearly identical during the full contact and rocking phases, with only a minor rocking component $v_{u 3 r}$. Finally, the rocking mode eigenvector represents a rigid body action of the structure as a whole, with negligible elastic components. This explains why the modified frequency parameter $p_{l}$, derived specifically for a rigid body, provides a good approximation for the rocking mode of the flexible structure.

In Figure 5, the predicted modal frequencies and compound shapes during full contact and rocking phases are compared to the experimentally obtained reference operational 
acceleration profiles. The operational acceleration profiles are empirical estimates for the mode shapes of the structure, obtained purely from the acceleration data recorded during free vibration tests. They are analogous to operational deflection shapes [24] and are identified from the frequency domain analysis of the recorded translational accelerations of individual rocking half-cycles [11]. As such, they describe the combined effect of the vibrations and rocking action experienced by each mode, similar to the compound mode shapes. The agreement between the mode shape estimates from the experiments and analytical simulation was quantified using the Modal Assurance Criterion (MAC) number. The MAC is a scalar constant which describes the consistency of two mode shape estimations [24]. In Figure 5, the MAC values are listed under a comparison of the experimentally identified and analytically predicted modal frequencies which are respectively shown in brackets. The good agreement between mode shape estimations validates the proposed modal analysis approach.
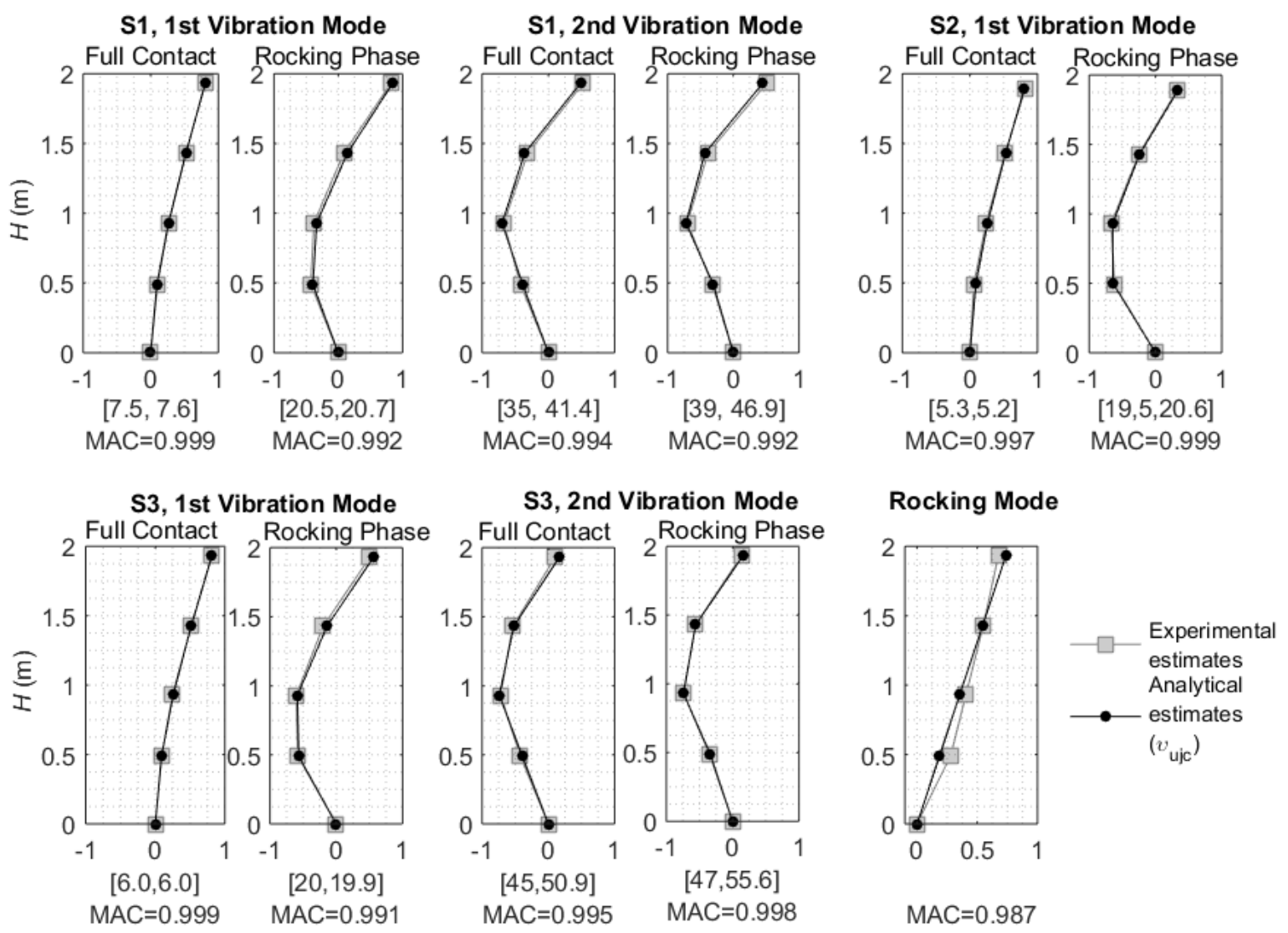

Figure 5. Comparison of analytical and experimental modal estimates for all specimens. Under each mode shape estimate, the experimental and analytical estimates for modal frequencies are respectively shown in brackets, along with the MAC number which describes the consistency of the mode shapes.

The results presented in this section demonstrate general trends. Emergence of a hyperbolic 'rocking mode' solution, which is influenced primarily by structural geometry and scale, helps identify structural parameters which are critical for rocking response. It is noteworthy that this mode is weakly coupled with elastic response. More significantly, the results demonstrate that the first two vibration modes interact strongly with rocking while the $3^{\text {rd }}$ vibration mode does not. Therefore, the assumption of 'higher vibration mode uncoupling' is not accurate for all higher modes. This result has significant implications as vibrations models coupled with rocking experience a change of vibration frequencies and mode shapes. Additionally, the coupling leads to excitation of vibration modes at impact (see Section 4.3). 
These findings are useful for the investigation of the nonlinear dynamic response discussed in the next section.

Table 2. Employed analytical impact models and their salient characteristics

\begin{tabular}{|l|c|c|c|}
\hline $\begin{array}{c}\text { Impact } \\
\text { Model ID }\end{array}$ & $\begin{array}{c}\text { Impact } \\
\text { Model }\end{array}$ & $\begin{array}{c}\text { Coefficients of } \\
\text { Restitution }\end{array}$ & $\begin{array}{c}C_{p} \\
(\mathrm{~kg} \mathrm{~m} / \mathrm{rad} / \mathrm{s})\end{array}$ \\
\hline CIM & Classical & Empirical, $r_{e}$ & - \\
\hline DD1 & Dirac-delta & Empirical, $r_{e}$ & 1750 \\
\hline DD2 & Dirac-delta & Mean, $\left(\overline{r_{e l}}, \overline{r_{e r}}\right)$ & 1750 \\
\hline DD3 & Dirac-delta & Empirical, $r_{e}$ & 3000 \\
\hline DD4 & Dirac-delta & Empirical, $r_{e}$ & 1000 \\
\hline DD5 & Dirac-delta & Random, $r$ & 1750 \\
\hline
\end{tabular}

\subsection{Free vibration response}

This section evaluates the models presented in Section 2 by simulating the free vibration tests. In particular, the two different impact models discussed in Section 2.4 will be investigated. Table 2 summarises the investigated impact model parameters. The classical impact model (CIM) with empirically defined coefficients of restitution is considered, along with the Dirac-delta (DD) impact model with varying parameters.

In the free vibration tests, the experimental models were displaced to a large rocking angle and then released with zero initial velocity. As an example, results from a large amplitude free vibration rocking test on $\mathrm{S} 1$ are presented in Figure 6. Here, the initial rocking angle is approximately 0.11 radians, which corresponds to one third of the slenderness angle $\alpha_{c g}$ of the specimen. In Figure 6, the experimental results are simulated using the impact models CIM and DD1. The former utilizes the classical impact model while the latter utilizes Dirac-delta forces to simulate impact. However, both models employ identical and impactspecific coefficients of restitution $r_{e}$ which were determined empirically using free vibration tests. By assuming that the total system energy is dominated by gravitational potential energy during large rocking motion, approximate estimates for the coefficient of restitution were obtained [11]. For rocking half-cycles with rocking amplitudes smaller than $0.01 \mathrm{rad}$, empirical coefficient of restitution estimates were not determined. In these cases, mean coefficients of restitution, determined separately for right and left corners of the experimental model $\left(\overline{r_{e r}}, \overline{r_{e l}}\right.$ in Table 1$)$, were utilized to model the response.

The first row of Figure 6 shows the rocking angle trace. Plotted also in this trace and the $2^{\text {nd }}$ row are the impact forces for DD1, shown with a light grey line. Before proceeding to compare different simulations and experiments, it is useful to take a closer look at these forces. From the top row, it can be observed that the impact forces are activated as the structure approaches $\theta=0$ and are deactivated once phase transition occurs. It is also noteworthy that the impact force activation angles become smaller for smaller rocking motion. For instance, for the first rocking half-cycle the impact force is activated at $\theta_{i}=0.01 \mathrm{rad}$ and for the $9^{\text {th }}$ rocking half-cycle this value is $\theta_{i}=0.004 \mathrm{rad}$. Additionally, the maximum amplitude of the impact force is observed to decrease for smaller rocking amplitudes. These observations are in accordance with the derivations in Section 2.4. 

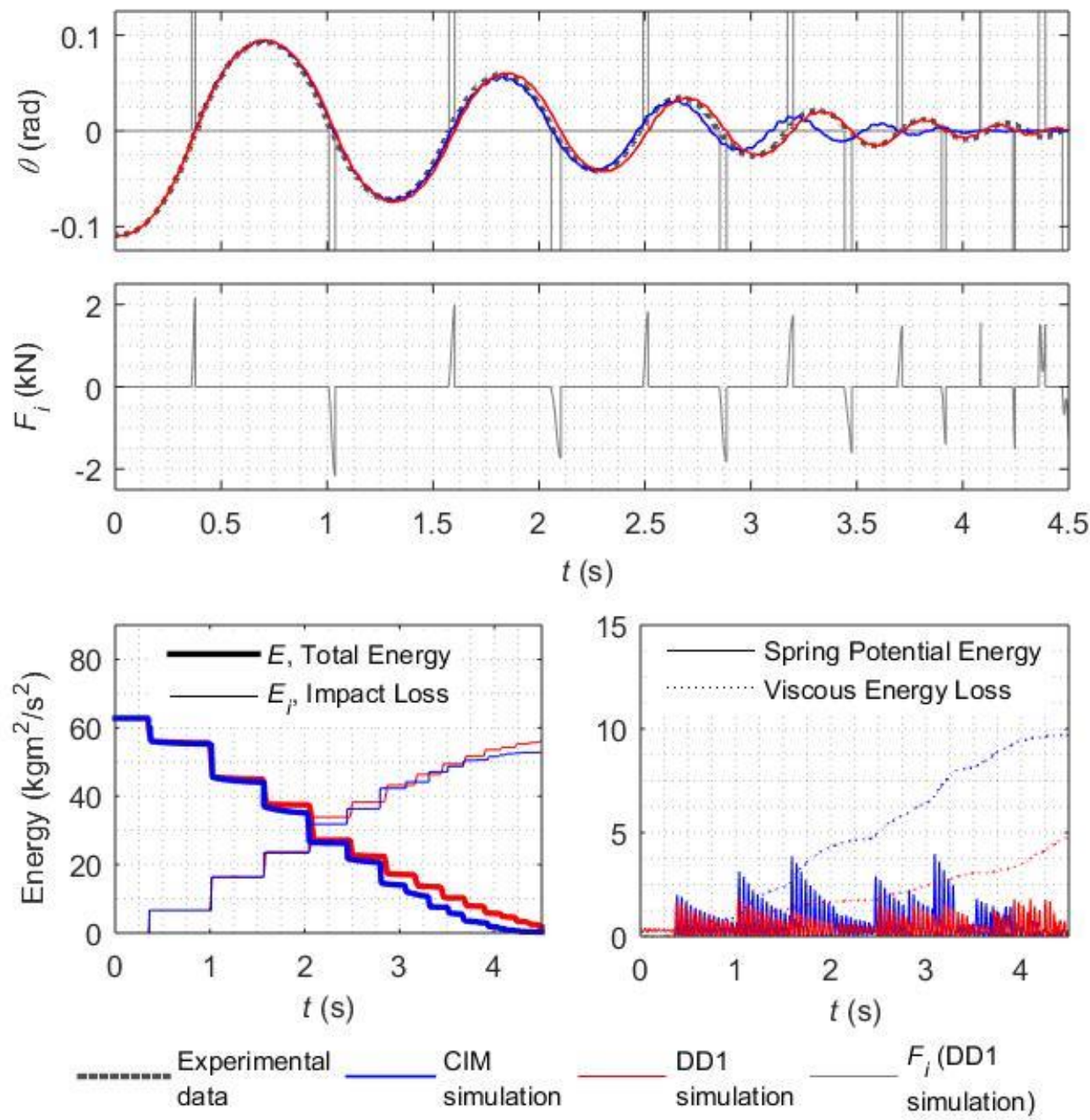

Figure 6. Comparison of a large free vibration test of $\mathrm{S} 1$ to simulations of two multi-mass analytical models: (first row) rocking angle, (second row) modelled impact forces, and (third row) comparison of the energy states during simulations.
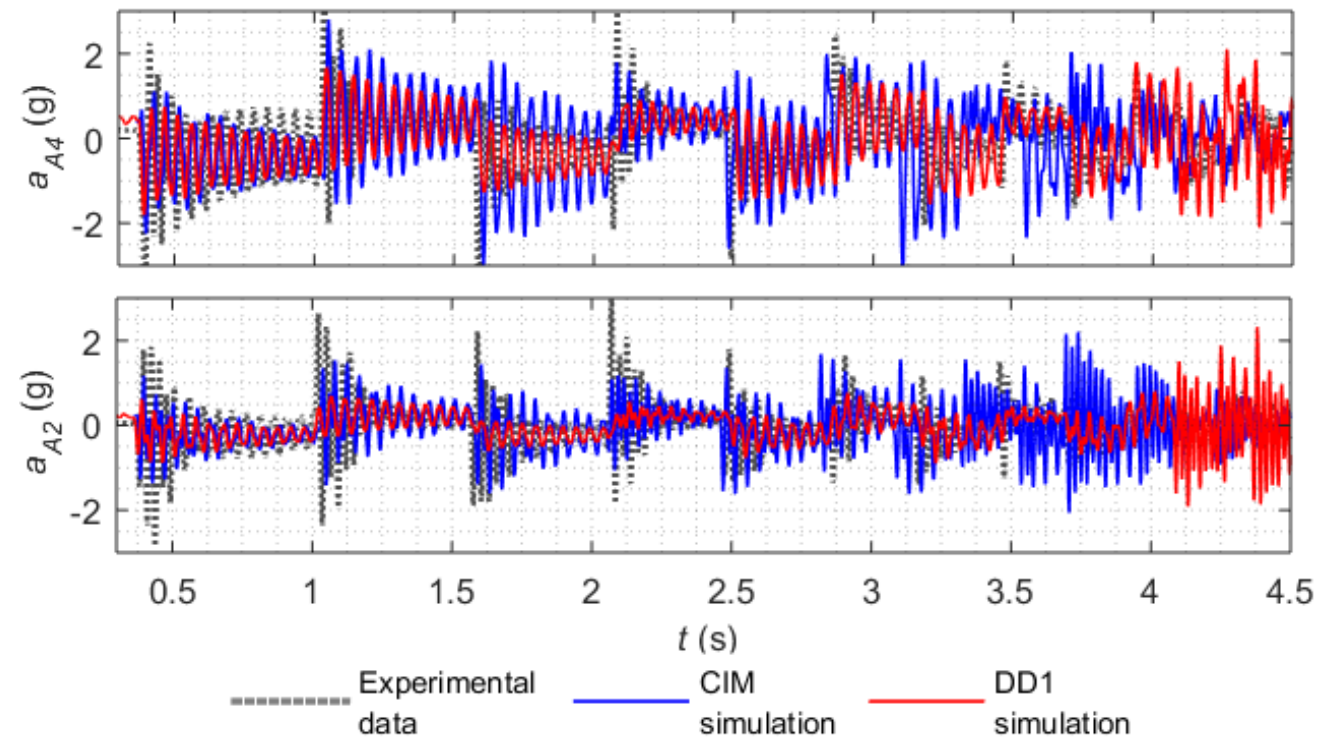

Figure 7. Comparison of a large free vibration test of S1 to simulations of two multi-mass analytical models: lateral accelerations at the (top row) top-height and (middle row) mid-height of the column 
In Figure 6 (top row), the results from both simulations compare well to the experimental rocking angle trace during the first few rocking half-cycles. The two traces start to differ perceptibly after $t=2.5 \mathrm{~s}$, where the classical impact model becomes less accurate than the Dirac-delta impact model. This is surprising as both models utilize the same modal damping values and coefficients of restitution. However, different impact models simulate different energy transfer to the elastic motion during impact. The energy graphs in Figure 6 (third row) indicate that when an instantaneous impact is assumed (CIM), the energy transfer to the oscillations tends to be higher, leading to an under-prediction of rocking motion and leading to more energy dissipation by viscous damping. Additionally, after $t=3 \mathrm{~s}$, the instantaneous energy dissipation in CIM led to several consecutive phase transitions within a single 'impact', leading to exaggerated disappearance of rocking motion. These several consecutive phase transitions, have been observed in the literature for other flexible rocking structure models employing classical impact assumptions (see [13,16-17]). They are a typical result of imparting excessive energy to vibrations at impact and are not observed experimentally. The Dirac-delta formulations seldom experience consecutive phase transitions and capture the rocking response with better accuracy.

Figure 7 compares the recorded and simulated lateral accelerations at the mid- and top-heights of the structure (denoted by $a_{A 2}$ and $a_{A 4}$ ). While neither simulation captures the acceleration response accurately for all half-cycles, both simulations capture the general characteristics of the acceleration data. The characteristic step function arises due to the rotational accelerations induced by gravity forces during rocking and was captured accurately by both models. Excitation of the $1^{\text {st }}$ and $2^{\text {nd }}$ uplifted mode at impact is also observed. The large magnitude of accelerations experienced by the classical impact model indicate significant energy transfer to vibrations at impact. The acceleration magnitudes are smaller for the Dirac-Delta model, which displays better agreement with experimental results before $t=4 \mathrm{~s}$.

It is noteworthy that after $t=3.5 \mathrm{~s}$, the superstructure vibrations are over-predicted by both models. The strong manifestation of accelerations at mid-height and their measured vibration frequency of approximately $40 \mathrm{~Hz}$, identifies them as accelerations due to the $2^{\text {nd }}$ uplifted mode. These large accelerations are a result of impact modelling assumptions which might not be valid for small amplitude rocking motion. In particular, it was observed that during the non-smooth transition from rocking to a full contact phase (see Equation 10), both models predict these unrealistic accelerations due to the excitation of the $2^{\text {nd }}$ vibration mode. This exemplifies an important modelling limitation.

The dependency of the simulation predictions on the impact model motivated a parametric study. In Figure 8, the rocking rotation and the mid-height acceleration response of various models listed in Table 2 are compared. The first row investigates the use of mean coefficients of restitution $\overline{r_{e l}}$ and $\overline{r_{e r}}$ (see Table 1) instead of utilizing empirically determined impact-specific values for the coefficient of restitution. In general, a good overall prediction is observed for DD2 as mean coefficients of restitution overpredict and underpredict energy dissipation for various impacts. Therefore, in the absence of impact-specific values for the coefficient of restitution, mean coefficients of restitutions can be utilized to simulate pulse and earthquake response. Next, in the $2^{\text {nd }}$ row of Figure 8, the effect of varying the Diracdelta constant $C_{p}$ was considered. As discussed in Section 2.4, larger values of $C_{p}$ (DD3) lead to impact forces of larger magnitude that are tightly concentrated around $\theta=0$. This causes a larger transfer of energy to the elastic motion compared to experiments, resulting in an under-prediction of rocking motion and an over-prediction of mid-height accelerations. In subsequent sections, an approximate value of $C_{p}=1750 \mathrm{~kg} \cdot \mathrm{m} / \mathrm{s}$ is utilized for all specimens. It is possible to define an impact-specific value for this parameter to accurately capture the 
acceleration response of each individual rocking half-cycle but this is outside the scope of this study.
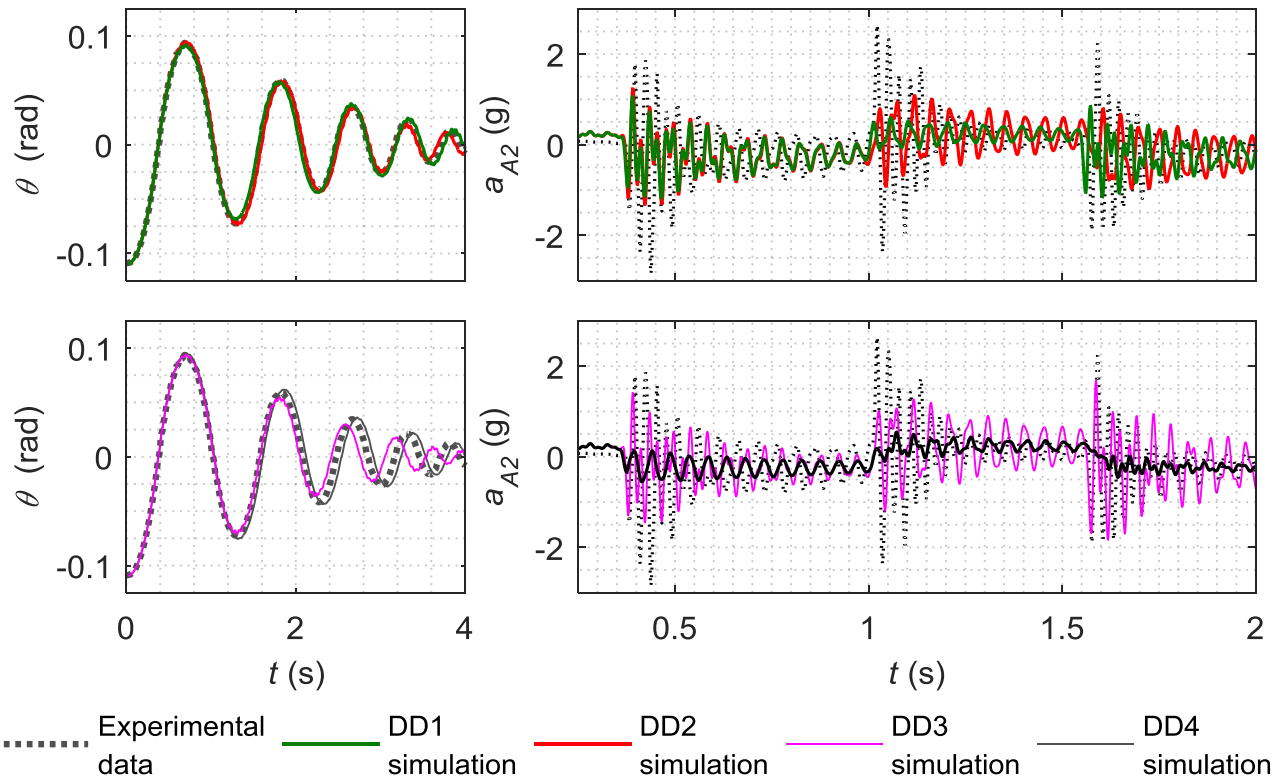

Figure 8. Comparison of the rocking rotation and mid-height acceleration traces of a large free vibration test of S1 to simulations using different models: (top row) impact-specific versus mean coefficients of restitution and (bottom row) different $C_{p}$ impact parameters.

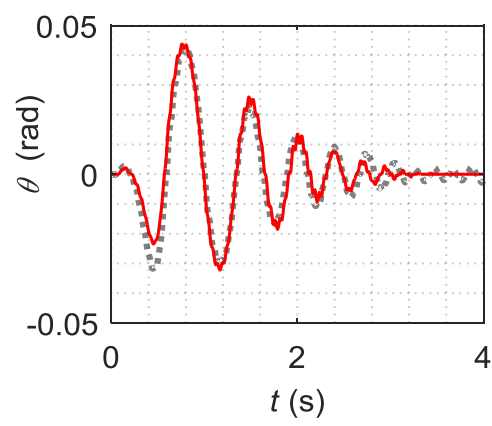

Experimental data

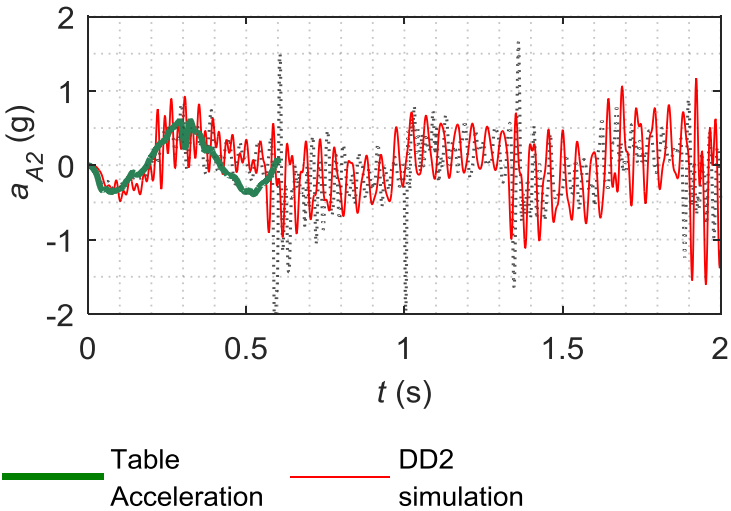

Acceleration simulation

Figure 9. Comparison of the rocking and mid-height acceleration traces of a pulse test on S1 to the corresponding traces simulated by the multi-mass analytical model.

\subsection{Pulse and earthquake excitation response}

In the previous section, the experimental free vibration test results were used to validate models and evaluate the impact modelling assumptions. The current section investigates the ability of the analytical model to recognize uplift conditions and to capture the ensuing rocking and acceleration response under pulse and earthquake excitations.

A phased and vertically translated cosine pulse was utilized in all pulse excitation tests. In Figure 9, the experimental rocking and mid-height acceleration responses from a pulse test on $\mathrm{S} 1$ (with pulse characteristics defined by frequency $\omega=15 \mathrm{rad} / \mathrm{s}$ and amplitude $A=0.4 \mathrm{~g}$ ) are compared to analytical simulations using the DD2 impact model (Table 2). The rocking behaviour and acceleration response are generally captured with good accuracy. More specifically, the maximum rocking motion is captured well, while the lack of precise estimates for the coefficients of restitution causes the accuracy of the rocking response simulation to slowly reduce. 
To generalize the results, it is useful to evaluate the analytical modelling predictions for the maximum rocking $\theta_{\max }$ for a range of pulse excitations. In Figure 10, rocking response spectra compare pulse experiments on S1 and S2 to the analytical model with impact model DD2 (see Table 2). The first row shows the rocking response spectra of pulses with varying frequency and fixed amplitude. In the second row, the response to pulses with a fixed frequency and various amplitudes is shown. Each test and simulation was repeated a minimum of two times. The markers in Figure 10 represent individual tests, while the lines represent mean values from the repeated tests. Generally, the analytical model provides a good prediction of maximum rotation. For both S1 and S2, the predictions lie within $10 \%$ of the experimental observations. The variance in repeated tests is small suggesting that even without precise information on coefficients of restitution, the direct amplification mechanisms induced by simple pulses can be simulated with good accuracy.
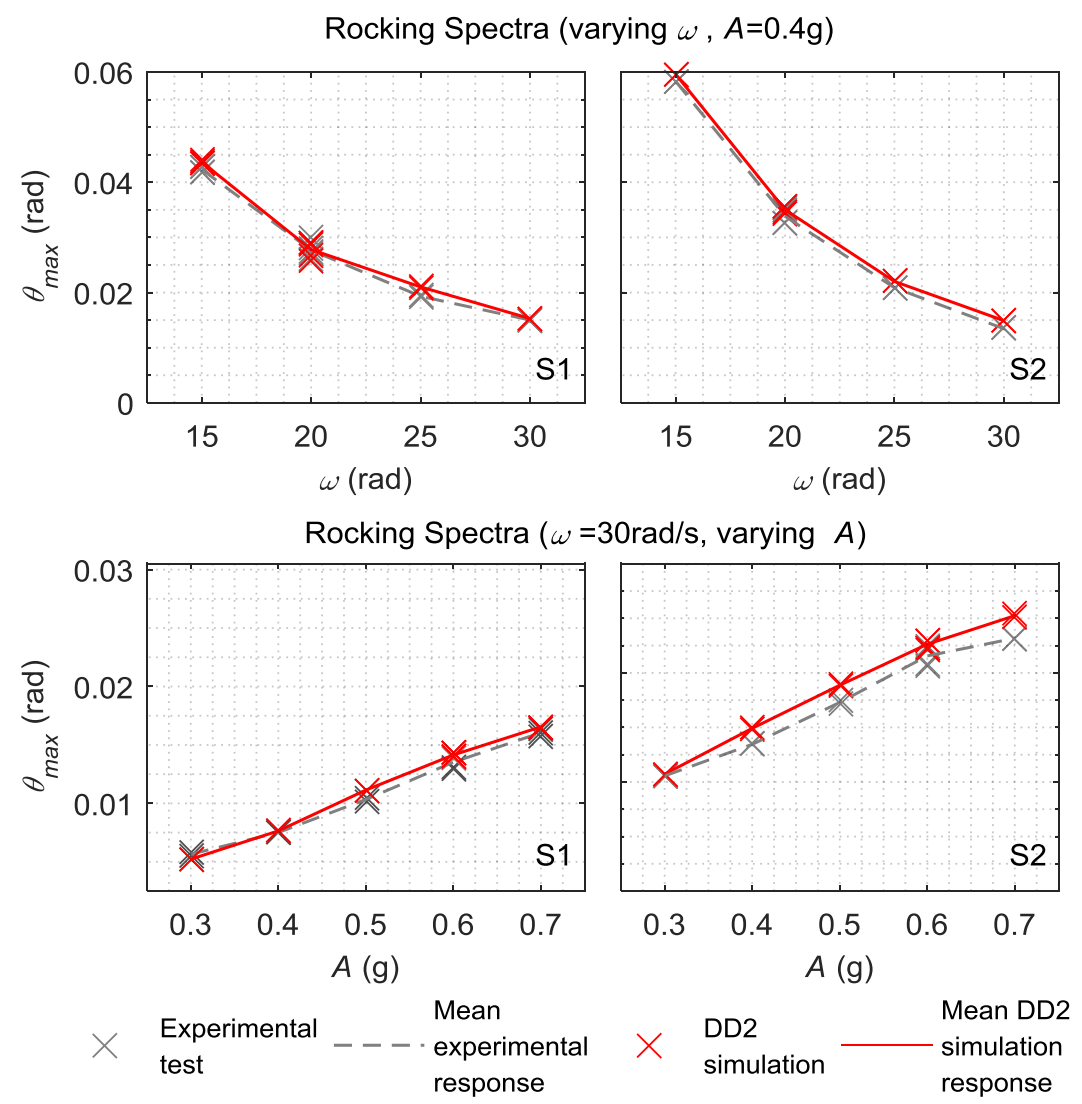

Figure 10. Comparison of the experimental rocking response spectra of S1 and S2 to the corresponding spectra simulated by multi-mass analytical models under the influence of pulse excitations.

To investigate the earthquake response experimentally, a single non-pulse type earthquake excitation, scaled to multiple amplitude levels, was considered. However, the experimental response to near-identical table excitations was observed to be variable. It was suggested that this variability is a result of variable energy dissipation and transfer to vibrations at impact [11]. It is therefore desirable to evaluate the ability of analytical simulations to capture similar response variability with different impact parameters.

Figure 11 shows the rocking and mid-height acceleration response to two amplitude scaled (ground motion scaling factor, $A_{s c}=1.1$ ) $1940 \mathrm{El}$ Centro record tests on S1. These tests (EC Test 1 and EC Test 2) resulted in drastically different response despite nearly identical table motions (see Figure 11, top row). To highlight the response differences, the ratio of the 
maximum rocking angle experienced during a test to the slenderness of the structure can be examined. This value is approximately 0.3 for EC Test 1 and only 0.03 for EC Test 2.
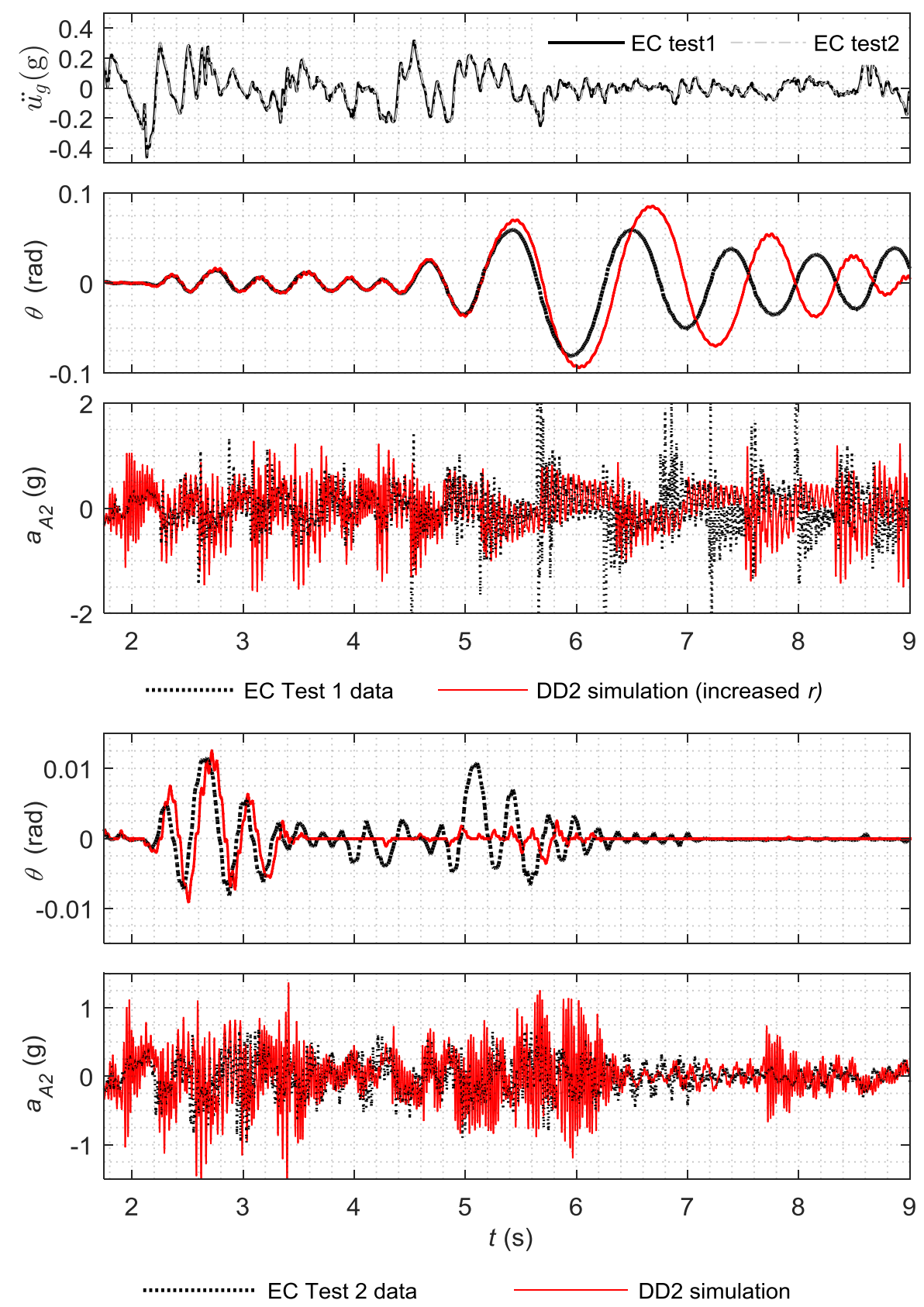

Figure 11. Comparison of the rocking and mid-height acceleration traces recorded during a repeated earthquake excitation test to the corresponding traces simulated by multi-mass analytical models.

To simulate EC Test 1 and EC Test 2, impact model DD2 was first used with the mean coefficients of restitution $\left(\overline{r_{e r}}, \overline{r_{e l}}\right)=(0.93,0.87)$. This resulted in a good prediction of the EC Test 2 results (Figure 11, fourth row). The simulations for the EC Test 1 with the same model were similar to EC Test 2 simulations, but did not agree with the EC Test 1 experimental results. However, by increasing the coefficient of restitution to $(0.97,0.91)$, the multi-mass model prediction of EC Test 1 improved dramatically (Figure 11, second row). However, after realization of larger amplitudes around $t=6 \mathrm{~s}$ the response was underdamped. Thus, the EC Test 1 results indicate that less energy was dissipated during smaller initial 
rocking half-cycles. Therefore, the initial small rocking response was not damped out in the experiment, and then the response was amplified dramatically. Contrastingly, this initial small amplitude motion was damped out too quickly with the original mean coefficients of restitution.

The acceleration predictions follow similar trends discussed earlier. The predictions using impact model DD2 capture the acceleration trace with reasonably good accuracy. However, the over-prediction of higher mode accelerations for small rocking motion is again observed. It is likely that this additional energy transmitted to superstructure vibrations contributes to errors in simulating the rocking response.

From Figure 11, it is clear that an exact evaluation of rocking response is difficult due to the sensitivity of the response. An alternative modelling strategy is to use a probabilistic distribution of coefficients of restitution to estimate the variability in energy dissipation [25], and therefore better capture the variability of earthquake response. To achieve this, the experimentally determined mean coefficients of restitution $\left(\overline{r_{e r}}, \overline{r_{e l}}\right)$ and their standard deviation $\sigma_{r}$ values (see Table 1) were used to assign a random coefficient of restitution for each impact. However, the differences in energy dissipation for small and large rocking motion were still not captured in the model.

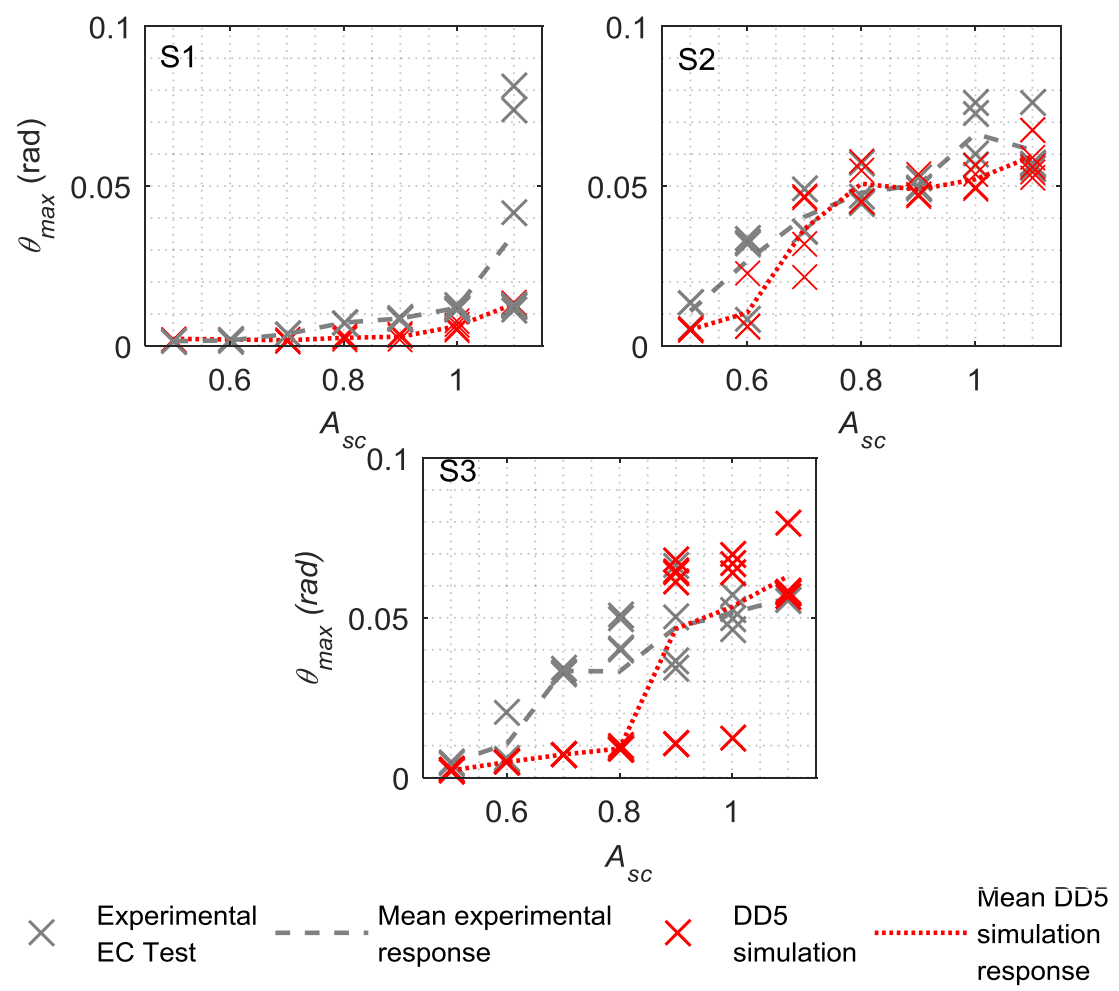

Figure 12. Comparison of the experimental rocking response spectra and the spectra simulated by multi-mass analytical models under the influence of amplitude scaled earthquake excitations.

In Figure 12, the rocking spectral response of all three experimental specimens to the scaled El Centro earthquakes is evaluated. Impact model DD5 (Table 2) was used with randomly generated coefficients of restitution. Each simulation was repeated a minimum of three times for each amplitude scale using the recorded table motions. From the individual simulation results for each amplitude scale, a mean spectrum was computed by averaging the rocking response.

In general, the variability in experimental and simulation results is similar, and the trend of increased rocking response with an increase in $A_{s c}$ is captured. However, the 
maximum rocking tended to be under-predicted. The primary discrepancy between modelling and experimental results, for all three specimens, occurs at the transition between relatively small and large rocking response at approximately 0.01 radians. This transition is smoother for experimental results, but more abrupt for simulation results. In this transition zone ( $A_{s c}=1.1$ for $\mathrm{S} 1, A_{s c}=0.6$ for $\mathrm{S} 2$, and $A_{s c}=0.7-0.8$ for S3), the simulation results are particularly under-predicted. Again, this is likely due to the inaccurate simulation of energy dissipation and transfer to vibrations during small rocking motion. As demonstrated in earlier work [26] and now in this study, the presence of initial small rocking motion at the onset of the large amplitude portions of the accelerogram may make a significant difference in altering the following response. Further refinement of the model impact parameters could be implemented to ensure that results are more conservative, but improvement of modelling impacts for small rocking motion would remain a necessity.

\section{CONCLUSIONS}

This paper proposes a new analytical model of a multi-mass rocking structure that addresses several limitations of earlier models. In addition, a modal decomposition method is introduced to explore the coupling of all vibration modes with rocking action. Evaluation of the ability of analytical models to capture the observed experimental results led to the following conclusions:

- The modal decomposition method successfully identified the dynamic characteristics of the rocking and vibration modes. Its specific application to the experimental specimens demonstrates that higher vibration modes can be coupled with rocking, resulting in different vibration characteristics and excitation mechanisms.

- The new Dirac-delta model allows the interaction of impact forces with structural vibrations. With this model, predictions for rocking rotation and acceleration response improved. However, there remains a need to improve the non-smooth transitions from rocking phases to full contact phases for better predictions.

- Correct estimation of the energy dissipation and transfer to vibration at impacts was critical for simulating the rocking and acceleration responses. This was achieved for large amplitude free vibration tests where empirical estimates of coefficient of restitution from experiments could be utilized.

- Response to pulse excitations was predicted successfully with multi-mass models. The use of precise coefficients of restitution was less important for maximum rocking predictions due to simple and dominant amplification mechanisms.

- Response to earthquake excitations was predicted with mixed success by the multi-mass models. The experimentally observed variability in maximum rocking response was partially captured by the analytical models when random coefficients of restitution were utilized. Limitations arose due to a systematic overestimation of energy dissipation for small rocking motion.

\section{REFERENCES}

[1] Huckelbridge AA, Clough RW. Seismic response of uplifting building frame. Journal of the Structural Division 1978; 104(8): 1211-1229.

[2] Sharpe RD, Skinner RI. The seismic design of an industrial chimney with rocking base. Bulletin of the New Zealand National Society for Earthquake Engineering 1983; 16(2): 98106. 
[3] McManus KJ. The seismic response of bridge structures free to rock on their foundations. University of Canterbury, 1980.

[4] Toranzo-Dianderas LA. The use of rocking walls in confined masonry structures: a performance-based approach. University of Canterbury, 2002.

[5] Pollino M, Bruneau M. Analytical and experimental investigation of a controlled rocking approach for seismic protection of bridge steel truss piers. Buffalo: 2008.

[6] Roke D, Sause R, Ricles JM, Chancellor NB. Damage-free seismic resistant self-centering concentrically-braced frames. vol. ATLSS Repo. Bethlehem: 2010.

[7] Wiebe L. Design of controlled rocking steel frames to limit higher mode effects. University of Toronto, 2013.

[8] Psycharis IN. Dynamics of flexible systems with partial lift-off. Earthquake Engineering \& Structural Dynamics 1983; 11(4): 501-521.

[9] Yim CS, Chopra AK. Simplified earthquake analysis of multistory structures with foundation uplift. Journal of Structural Engineering 1985; 11(12): 2708-2731.

[10] Acikgoz S, DeJong MJ. The rocking response of large flexible structures to earthquakes. Bulletin of Earthquake Engineering 2013; 12(2): 875-908.

[11] Acikgoz S, Ma QT, Palermo A, DeJong MJ. Experimental identification of the dynamic characteristics of a flexible rocking structure. Journal of Earthquake Engineering. In press.

[12] Guyan RJ. Reduction of stiffness and mass matrices. AIAA Journal 1965; 3(2): 380.

[13] Acikgoz S, DeJong MJ. The interaction of elasticity and rocking in flexible structures allowed to uplift. Earthquake Engineering \& Structural Dynamics 2012; 41(15): 2177-2194.

[14] Acikgoz S, DeJong MJ. Analytical and experimental observations on vibration modes of flexible rocking structures. Society of Earthquake and Civil Engineering Dynamics Young Engineers Conference, Newcastle, UK: 2013.

[15] Vassiliou MF, Mackie KR, Stojadinovic B. Dynamic response analysis of solitary flexible rocking bodies: modeling and behavior under pulse-like ground excitation. Earthquake Engineering \& Structural Dynamics 2014; 43(10): 1463-1481.

[16] Truniger R, Vassiliou MF, Stojadinovic B. An analytical model of a deformable cantilever structure rocking on a rigid surface: experimental validation. Earthquake Engineering \& Structural Dynamics 2015; 44(15): 2795-2815.

[17] Vassiliou MF, Truniger R, Stojadinovic B. An analytical model of a deformable cantilever structure rocking on a rigid surface: development and verification. Earthquake Engineering \& Structural Dynamics 2015; 44(15): 2795-2815.

[18] ElGawady MA, Ma QT, Butterworth JW, Ingham JM. Effects of interface material on the performance of free rocking blocks. Earthquake Engineering \& Structural Dynamics 2010; 40(4): 375-392.

[19] Prieto F, Lourenço PB, Oliveira CS. Impulsive Dirac-delta forces in the rocking motion. Earthquake Engineering \& Structural Dynamics 2004; 33(7): 839-857.

[20] Shampine L, Gladwell I, Thompson S. Solving ODEs with MATLAB. Cambridge: Cambridge University Press; 2003.

[21] Palmeri A, Makris N. Linearization and first-order expansion of the rocking motion of rigid blocks stepping on viscoelastic foundation. Earthquake Engineering \& Structural Dynamics 2008; 37(3): 1065-1080.

[22] Caughey TK, O'Kelly MEJ. Classical normal modes in famped linear dynamic systems. Journal of Applied Mechanics 1965; 32(3): 583-588.

[23] Foss KA. Coordinates which uncouple the equations of motion of damped linear dynamic systems. Massachusetts Institute of Technology, MIT-TR-25-20. Boston: 1956.

[24] Ewins DJ. Modal testing: theory, practice and application. Baldock: Research Studies Press; 2000.

[25] Liberatore D, Spera G, Alessandro GD, Nigro D. Rocking of slender block subjected to seismic motion of the base. Proceedings of the 12th European Conference on Earthquake Engineering, London: 2002.

[26] DeJong MJ. Amplification of rocking due to horizontal ground motion. Earthquake Spectra 2012; 28(4): 1405-1421. 